\title{
VEJEZ, INTERGENERACIONALIDAD Y CIUDADANÍAS. LAS PRÁCTICAS CORPORALES EN UN CLUB DE VIDA DE MEDELLÍN (COLOMBIA) ${ }^{1}$
}

\author{
OLD AGE, INTERGENERATIONALITY AND CITIZENSHIP: CORPORAL PRACTICES AT A LIFE \\ CLUB IN MEDELLÍN (COLOMBIA)
}

\section{VELHICE, INTERGENERACIONALIDADE E CIDADANIAS. AS PRÁTICAS CORPORAIS EM UM CLUBE DE VIDA DE MEDELLIN (COLÔMBIA)}

Paola Andrea García Peláez ${ }^{2}$

\section{Resumen}

\begin{abstract}
Este artículo hace parte de la investigación "Las ciudadanías en un grupo de adultos mayores: una mirada al club de vida Mi Segundo Hogar" (Universidad de Antioquia), la cual permitió comprender los aportes que hace un club de vida de la ciudad de Medellín al proceso vital humano de la vejez, de las relaciones intergeneracionales, las ciudadanías y el campo de interacciones que promueven las prácticas corporales allí realizadas. Se privilegió, así, la investigación etnográfica, utilizando como instrumentos la observación participante, las entrevistas y los diarios de campo. Entre los resultados obtenidos podemos decir que el club de vida se convierte en un espacio potenciador y revitalizador de las capacidades de los adultos mayores, allí está su posibilidad de seguirse construyendo como ciudadanos mediante el fomento de las relaciones con diferentes grupos de edades y con la oportunidad de seguir construyendo propuestas a favor de la sociedad.
\end{abstract}

Palabras clave: vejez; intergeneracionalidad; ciudadanías; prácticas corporales

Abstract

This article is part of the research titled "Citizenship in a group of older adults: a look at Mi Segundo Hogar life club" (Universidad de Antioquia), which led to understanding the contributions of a life club in the city of Medellín to the human vital process of old age, intergenerational relations, citizenships and the field of interactions promoting physical activity there. Thus, privilege was given to ethnographic research, using participant observation, interviews and field journals as instruments. The results obtained show that life clubs become areas for enhancing and revitalizing the physical aptitudes of the elderly, granting them the possibility to continue growing as citizens by fostering relationships with different age groups and with the opportunity to continue constructing proposals in favor of society.

Keywords: old age; intergenerationality; citizenships; physical activity

1 Artículo asociado a la investigación "-Las ciudadanías en un grupo de adultos mayores: una mirada al club de vida Mi Segundo Hogar”.

2 Aspirante a magíster en Salud Colectiva. Universidad de Antioquia. Correo electrónico: deandrea23 @gmail.com 
Este artigo faz parte da pesquisa "As cidadanias em um grupo de idosos: um olhar ao clube de vida Meu Segundo Lar" (Universidade de Antioquia), que permitiu compreender as contribuições feitas pelo clube de vida na cidade de Medellin ao processo vital humano da velhice, das relações intergeneracionais, as cidadanias e o campo de interações promovidas pelas práticas corporais realizadas. Adotou-se o método de investigação etnográfica, utilizando como instrumentos a observação participante, as entrevistas e os diários de campo. Entre os resultados obtidos, é possível afirmar que o clube de vida é um espaço energizante e revitalizante das capacidades dos idosos; neste espaço eles têm a possibilidade de se construir como cidadãos por meio das relações fomentadas com diversos grupos de idades e com a chance de continuar construindo propostas benéficas para a sociedade.

Palavras chave: velhice; integeneracionalidade; cidadanias; práticas corporais

Fecha de recepción: 8 de junio de 2016

Fecha de aprobación: 12 de diciembre de 2016

Para citar este articulo:

García, P. (2017). Vejez, intergeneracionalidad y ciudadanías. Las prácticas corporales en un club de vida de Medellín (Colombia). Lúdica Pedagógica, (25), 137-144. 


\section{INTRODUCCIÓN}

Con el incremento del envejecimiento en la población mundial surge la preocupación por asuntos como la salud, situación económica, nutricional, de empleo, participación, entre otras, de este grupo poblacional. Inicialmente, las Naciones Unidas en 1969, dictan una serie de resoluciones, y posteriormente, en 1982 se realiza en Viena la primera Asamblea Mundial sobre Envejecimiento, centrado en mejorar el sistema pensional y de salud de las personas mayores (Barrera, 2011). -Veinte años después, en 2002, se realiza la Segunda Asamblea Mundial de Naciones Unidas sobre Envejecimiento, y se configura como instrumento para la creación de políticas relacionadas con este tema, haciendo tres recomendaciones fundamentales: relación entre personas mayores y desarrollo, fomento de salud y bienestar durante toda la vida y, por último, la creación de entornos propicios y favorables (Imserso, 2007).

Ahora bien, en Medellín, donde se llevó a cabo esta investigación, se ha venido evidenciando, al igual que en otras ciudades y países, un incremento de la población adulta mayor pasando de $94.651(5,16 \%)$ a 148.052 (5,92 \%) para 2005 (Municipio Medellín, 2006). Dicha situación propició el camino para crear la Política Municipal de Envejecimiento y Vejez, hoy vigente en Medellín. De igual forma se dio inicio a la conformación de los clubes de vida como una directriz de la Presidencia de la República en los años 1990. Al respecto, una de las participantes tuvo la oportunidad de estar en esta iniciativa y relata:

"La Consejería de la Presidencia para la Familia invitó a 14 ciudades del país a que estuvieran en la iniciativa de conformar los clubes de vida. Alrededor de los centros de salud había un grupo de hipertensos y esos eran los grupos de la tercera edad que se veía en algunos barrios y nos invitaron a aplicar un modelo de España que era "El centro vida", las características y funcionamientos, los recursos y demás, en el momento no lo teníamos disponible como tal, como Centro Día, entonces decidimos llamarlos Club de Vida, un espacio físico donde las personas mayores van y se reúnen de acuerdo con un interés personal para compartir una actividad, bien sea de salud, recreativa, o de capacitación no formal o de ocupación del tiempo libre en manualidades, en danza en teatro, actividades recreativas y culturales." (EN 10, M-“LAB”) ${ }^{3}$.

3 Codificación dada a las entrevistas.
Fue así como en Medellín, durante la administración de los años 1990-1992, la Secretaría de Bienestar Social, como entidad estatal, puso a disposición de la comunidad mayor de 50 años de edad, la asesoría y dotación para el montaje de los clubes de vida; de esta forma comenzaron su actividad muchos de ellos y, aún hoy, permanecen en distintos sectores de la ciudad. El tipo de actividades que se desarrollan en estos escenarios están direccionados hacia prácticas corporales diversas como el baile, yoga, musicoterapia, entre otros.

Ahora bien, según los planteamientos expuestos, es necesario entender que con la especificidad de cada periodo histórico y de cada cultura, la vejez ha sido vista como una etapa de la vida donde las personas pueden ser sujetos de respeto o de desprecio y burla. Esto constata la idea de que estas maneras de ver el envejecimiento no pueden estar desligadas de un contexto social, de una cultura, de una época, de las formas de interactuar, y de las formas como circula y se distribuye la riqueza en una sociedad, en síntesis, de cómo nos relacionamos con nosotros, con la naturaleza y con la humanidad en general. Por ello, el envejecimiento, "como parte del proceso vital humano no puede estar ligado a realidades estáticas, sino que son realidades interarticulares, en movimiento constante y que precisa para su comprensión de acercamientos interdisciplinares y de transformatividad permanente" (Franco, 1999).

Pensar asíla vejez es lo que conduce a que en esta investigación se haya optado más que por la categoría de ciudadano, por la de ciudadanías, en el sentido que le atribuye

Cusicanqui, como posibilidad de realización de lo diferente en contextos caracterizados por la diversidad. A la manera de un rescate de las sociedades abigarradas, esto es, de unidad en la diferencia. "Hay que pluralizar la palabra ciudadanía, hay que hablar de ciudadanías. Porque, de hecho, hay modos diferentes de ser y sentirse ciudadana o ciudadano y hay una larga experiencia de prácticas democráticas diferenciadas" (Rivera, 2005, p. 4).

En este mismo sentido de pensarse en el rescate de lo diferente para apropiarnos del conocimiento que las prácticas corporales suscitan, se hace sugerente idearlas como ese momento potencial para el establecimiento de redes de encuentro, ya sea con el otro o consigo mismo. "Por lo tanto, es preciso estar atento no solo a la práctica, sino más bien al campo de relaciones que promueve" (Soares y Carvalo, 2010). Teniendo presente lo anterior, 
se puede decir que las prácticas corporales son también prácticas sociales, lo cual permite trascender una visión enmarcada de ellas como la simple ejecución de actividades o ejercicios físicos ahistóricos y universales. Las prácticas corporales son también dinámicas, cambiantes y rebasan una visión instrumental, están ancladas en un campo de actuación donde se juegan intereses y tensiones que podrían generar o inhibir la construcción de nuevas prácticas, saberes y ciudadanías en la población adulta mayor.

\section{METOdOLOGÍA}

Se optó por la investigación cualitativa, y el método etnográfico, que se entiende desde Joyceen Boyle (2003) como aquella observación directa que propicia luces sobre un grupo de personas y que brinda la posibilidad de ver el mundo a través de su óptica. La aproximación se realizó desde el enfoque fenomenológico, que al decir de Minayo (2009), procura entender los fenómenos sociales desde la perspectiva de los actores, las personas crean una intersubjetividad con los otros que da forma a la existencia social, donde la presencialidad y la relación cara a cara son determinantes.

La construcción del informe se hizo a partir de una serie de visitas al club de vida donde participaban los adultos mayores, y con base en ello se utilizó el diario de campo, que como afirma Sandoval (1997), es la herramienta fundamental en la etnografía, y la observación como participante, que, al decir de Galeano (2004), se hace posible cuando el investigador revela a los participantes su interés por ellos dentro del ejercicio investigativo y enfatiza la observación sobre la participación, y el diario de campo para plasmar los observado. También se realizaron entrevistas semiestructuradas (Ander-Egg, 1995), y una revisión documental como apoyo fundamental para la comprensión del objeto de estudio que estuvo centrado en contribuir a la comprensión de los aportes que hace el Club de Vida Mi Segundo Hogar, de Medellín, a los procesos de construcción de ciudadanías de un grupo de adultos mayores que en él participa.

Por último, para el análisis de la información y alcanzar validez y confiabilidad, que aluden al grado de coherencia y lógica interna, se triangularon las entrevistas semiestructuradas, observaciones y revisión documental, estrategia que permite, como afirma Galeano
(2004), la evaluación de la consistencia de los datos contrastándoles, es decir, confrontando lógicas, saberes, lecturas de la situación y versiones sobre el tema de interés del estudio.

\section{RESULTADOS}

La participación de los adultos mayores (AM) puede ser muy diversa, esta se concreta en espacios o actividades determinadas, donde los intereses o motivos para que se lleven a cabo, también lo sean, además de que están permeadas por aspectos como el sexo, el estado civil, el nivel educativo, entre otras, que coadyuvan a esa diversidad. Para este caso existe un lugar concreto: el club de vida Mi Segundo Hogar, el cual se convierte en un campo, entendido desde Bourdieu (2000), como aquel espacio social dinámico y estructurado donde existen jerarquías y reglas de juego propias, y donde se va configurando una red de relaciones.

\section{"Como un estorbo, obsoletos y cansones": algunas concepciones sobre vejez}

Los adultos mayores también se miran a sí mismos mediante un acto reflexivo que les posibilita autopercibirse, por ello se les preguntó sobre cómo pensaban que los veía o los percibía la sociedad en general, y refirieron al instante los sentimientos de rechazo y relegamiento, de poco respeto y desconocimiento de la experiencia que portan consigo.

Es una especie de muerte social que se declara cuando al AM se le interna en un centro de protección social, y se le distancia de las cosas que aman, cuando se les lleva forzosamente a la inactividad, cuando llegada la jubilación, se le arranca compulsivamente del círculo de sus relaciones sociales (Rodrigues, 2006). Al respecto una participante nos dice: "Ya usted a partir de los 60 años ya es obsoleto, ya no sirve para nada; ¿quién dijo que uno porque tenía 60 años no podía dar?" (ENT. 03 M.NAN). "Sencillamente los viejos estorbamos, a un ancianato nos vamos de una" (ENT 03 M "NAN" AD).

Sin duda, adjetivos como obsoletos, estorbosos, entre otros, han posibilitado que haya un quiebre en las posibilidades de seguir activos en la sociedad de una u otra manera. Es paradójico que nuestra cultura, mientras multiplica los viejos, al mismo tiempo los torna inútiles, improductivos y relativamente consumidores débiles o pasivos: 
Sus saberes y su experiencia no son más útiles a la construcción de progreso de la sociedad occidental, que cambiando continuamente los torna obsoletos y prescindibles ante las técnicas modernas que pueden ser cada vez más dominadas por jóvenes especialistas que saben más y más sobre menos y menos. (Rodrigues, 2006, p. 193)

Las relaciones intergeneracionales: "Si usted supiera y yo pudiera, el mundo sería distinto"

Las relaciones intergeneracionales pueden pensarse como aquellas que se dan entre generaciones que se relevan unas a otras, determinadas por aspectos demográficos, económicos, políticos, etc., de cada momento histórico, por tanto van cambiando, se van transformando. La investigadora Gomila (2005) afirma que "el intercambio de ayuda y el tipo de relaciones entre las generaciones ha tomado otras formas, sin suponer, sin embargo, un debilitamiento de dichas relaciones" (p. 510). Por ello al acercarse al tema de las relaciones intergeneracionales, es interesante pensarlas no solo como aquellas coordinadas y funcionales, sino también en su potencial conflictivo y divergente (Donati, 1999).

Iniciando porque la cultura, la juventud actual, no, es totalmente distinta al adulto mayor, como en la forma de vestir, como en la forma de alimentarse, como en la forma de los credos religiosos, la música; entonces se forma una brecha, no se pueden comunicar, entonces se generan los conflictos. (ENT 10 M “LA AB"-FUN).

Cuando en las entrevistas se les preguntaba a los adultos mayores sobre las relaciones con los otros grupos de edad, se refirieron con mayor énfasis al grupo de los jóvenes; existe allí una especie de grieta, de ruptura a la hora de relacionarse con ellos, creen que hay un mayor distanciamiento a la hora de entablar algún tipo de comunicación: "Lo que pasa es que los jóvenes y los niños no se sientan a conversar con los adultos mayores porque les parece que somos ridículos" (ENT 05 M "REI" AD). Sin embargo, se debe tener presente que, como afirma Romero (2009), "las diferencias dadas por el tiempo de vida o por la perspectiva generacional no pueden ser pensadas sin considerar las formas particulares en que converge el acceso a los bienes materiales y simbólicos alcanzados por cada grupo" (p. 62). Por lo tanto resultaría complejo discutir el proceso de diálogo y de relaciones intergeneracionales sin tener presente lo complejo de la creación de esas formas particulares.
El panorama expuesto deja entrever lo complejo que se pueden tornar las relaciones entre unas y otras generaciones, las contraposiciones que surgen entre adultos y jóvenes y lo influenciado que pueden llegar a estar por las dinámicas sociales y los contextos particulares; sin embargo, no se pueden generalizar que estas relaciones vayan siempre en contraposición, también pueden surgir allí ejemplos de solidaridad. Los adultos con sus narraciones nos dejan ver que se debe dar un compromiso social, de todos, no exclusivamente al interior de la familia, para que en la medida de lo posible no se rompan los lazos de comunicación con las otras generaciones, porque es allí donde se transmiten saberes que permiten la pervivencia de una cultura.

\section{"La gimnasia": una excusa para el encuentro}

Los clubes de vida, comprendidos como aquellos lugares donde los adultos mayores llegan para realizar algún tipo de actividad, sobre todo enfocados en programas de ejercicios, cobran otros valores en su vidas, y de ello dan cuenta las narraciones que los AM hacen sobre su participación en el club de vida y de las actividades que allí realizan; escucharlos es comprender un poco la satisfacción que encuentran en aquel lugar y lo que allí converge, pues es un espacio que abre diversas posibilidades. Quizás por ello hacer la gimnasia, como la llaman ellos, es el pretexto para lo que realmente subyace allí, esto es: una conversación, encontrarse con el otro, escuchar y ser escuchado, compartir experiencias, debatir, reír, jugar, cantar, adquirir nuevos conocimientos y, como una de las principales actividades, bailar; todas actividades que seguramente en muchos de sus hogares, les son vedadas. Este espacio grupal se convierte así en una fortaleza donde se potencia las ciudadanías pues se está consolidando su pertenencia a la sociedad.

Por ello algunos AM hablan de cómo gran parte de su tiempo lo dedican a estar en este lugar: "Como yo ya no tengo obligaciones en la casa, mi vida transcurre en el club, entonces me puedo venir a estarme de ocho a doce y de dos a seis sin ningún problema, esto tiene un atractivo especial" (ENT 06 M "REI" AD). Este relato nos podría llevar a afirma que con la participación de los AM en estos grupos de encuentro y convivencia hay un traslado en sus vidas de los escenarios de participación (y hasta en algunos de ellos de importancia), es decir, ya no es su centro de atención su hogar o el lugar donde laboraron, ya es el club de vida la estructura que incentiva y seduce hacia la participación social, es allí 
donde se pueden trazar cambios significativos para la vida de muchos de ellos, como lo afirma Souza (1987): "La participación en sí, como proceso social, cambia a sus propios actores, potencializa los grupos desfavorecidos, y hace crecer la confianza en sus propias capacidades y contribuye para su articulación" (p. 26) .

Razones como las expresadas por esta participante, son las que sustentan por qué hablar de prácticas corporales y no de actividad física. Las prácticas corporales, como advierte Carvalho (2007), "son modos de expresión, los cuales cargan sentidos y significados que las personas les atribuyen, [...] aquí hay una contraposición con la actividad física, que homogeniza el colectivo, estandariza e iguala el cuerpo con base en una racionalidad biomédica"(p.13). Las prácticas corporales por tanto se constituyen en un acontecimiento creador de nuevas formas de pensar, de expresarse, de actuar y de asumir este ciclo vital de una manera crítica y propositiva, no como estado de decadencia y flaquezas.

\section{CONCLUSIONES}

Se identifica entonces, como la adjetivación negativa a un ciclo vital humano que circula en la sociedad se hace cada vez más fuerte, situación paradójica en una sociedad que pregona constantemente sus avances en materia de innovación e inclusión, pareciese que una fuese en detrimento de la otra, además que muchos AM asumen como ciertos esos calificativos de decadencia, quietud, estorbosos, improductivos, entre otros, yendo así en quebranto de la construcción de ciudadanías. Lo que hay es una tarea grande por transformar en las culturas actuales y las venideras esas miradas negativas sobre la vejez.

De otro lado, en las relaciones intergeneracionales, pueden surgir contraposiciones entre adultos y jóvenes, las cuales muchas veces están influenciadas por las dinámicas sociales y los contextos particulares, sin embargo no se pueden generalizar que estas vayan siempre en contraposición, también pueden surgir allí ejemplos de solidaridad, porque si bien es cierto que esta época que estamos viviendo se caracteriza por un crecimiento acelerado de la población, por la cantidad de información que nos llega de diversos medios de comunicación, por cambios tecnológicos acelerados entre otros, los adultos con sus narraciones nos dejan ver que se debe dar un compromiso social, de todos, no exclusivamente en el interior de la familia, para que en la medida de lo posible no se rompan los lazos de comunicación con las otras generaciones, porque es allí donde se transmiten saberes que permiten la pervivencia de una cultura.

Se reitera una vez más que las razones o motivos por la cuales los AM asisten a los clubes de vida, están expresadas por la necesidad de sentir compañía, conversar, escuchar y ser escuchados, son lugares donde se sienten activos y que pertenecen a algo, trascienden el hecho de ir a realizar ejercicio como muchos lo llaman. Estos espacios, por tanto, deben ser pensados como potenciadores o revitalizadores de las capacidades de los adultos mayores, allí está la posibilidad de seguirse construyendo como ciudadanos, mediante el fomento de las relaciones con sus iguales, con otros grupos de edades, y con la oportunidad de construir propuestas a favor de toda la sociedad.

\section{REFERENCIAS}

Ander-Egg, E. (1995). Técnicas de investigación social. 24a. ed. Buenos Aires: Grupo Editorial Lumen.

Barrera, L. (2011). Demografía: el adulto mayor en la estructura poblacional. En: M. Quintero (comp.). La salud de los adultos mayores: una visión compartida (pp. 23-36). 2a. ed. Washington, DC: Organización Panamericana de la Salud.

Bourdieu, P. (2000). Cuestiones de Sociología. Madrid: Ediciones Istmo.

Boyle, J. (2003). Estilos de etnografía. En: J. Morse (ed.). Asuntos críticos en los métodos de investigación cualitativa. Medellín: Universidad de Antioquia, Facultad de Enfermería.

Carvalho, Y. (2007). Práticas corporais e comunidade: um projeto de educação física no Centro de Saúde Escola Samuel B. Pessoa. En: A. Braga (ed). Educação física e saúde coletiva: políticas de formação e perspectivas de intervenção. Porto Alegre: Editora da UFRGS.

Donati, P. (1999). Familias y generaciones. Desacatos, 2 , $1-24$.

Franco, S. (1993). Proceso vital humano. Proceso salud enfermedad: una nueva perspectiva. Conferencia presentada en el seminario marco conceptual para la formación de profesionales de la salud, Universidad Nacional.

Galeano, M. (2004). Estrategias de investigación social cualitativa: el giro de la mirada. Medellín: La Carreta.

Galeano, M. (2011). Diseño de proyectos en la investigación cualitativa. Medellín: Fondo Eeditorial Universidad Eafit. 
Gomila, M. (2005). Las relaciones intergeneracionales en el marco de la familia contemporánea: cambios y continuidades en transición hacia una nueva concepción de la familia. Historia Contemporánea, 31, 505-542.

Instituto de Mayoresy Servicios Sociales (Imserso) (2011). Relaciones Intergeneracionales. En: Envejecimiento Activo Libro Blanco, 565-596. Madrid: Ministerio de Sanidad, Política Social e Igualdad Secretaría General de Política Social y Consumo Instituto de Mayores y Servicios Sociales.

Municipio de Medellín (2006). Documento técnico de soporte POT. Recuperado el 28 de febrero de 2015 de http://www.medellin.gov.co/irj/go/km/ docs /wpccontent/Sites / Subportal\%20del\%20 Ciudadano/Plan\%20de\%20Desarrollo/Secciones / Informaci\%C3\%B3n\%20General/Documentos/POT/ medellinPoblacion.pdf

Minayo, M. (2009). La artesanía de la investigación cualitativa. Buenos Aires: Lugar Editorial.
Rivera, S. (2005). Un diálogo sobre las prácticas democráticas. Ciudadanías se escribe en plural. Recuperado el 24 de febrero de 2015 en: https:// es.scribd.com/document/91413240/Un-dialogosobre-la-diversidad-de-las-practicas-democraticasSilvia-Rivera-Cusicanqui

Rodrigues, J. (2006). Tabu da morte. 2 ed. Río de Janeiro: Editoria Fiocruz.

Romero, X., Ruiz, E.y Brigeiro, M. (2009).Haciauna sociedad para todas las edades: experiencias latinoamericanas de relaciones intergeneracionales. Santiago de Chile: Red Latinoamericana de Gerontología.

Sandoval, C. (1997). Investigación cualitativa. Bogotá: Corcas Editores.

Soares, F. y Carvalho, Y. (2010). Práticas corporais e grande saúde: um encontró possível. Ensaios, 16(4), 229-244.

Souza, M. (1987). Desenvolvimento de comunidade e participação. 2a. ed. Sao Paulo: Cortez. 